\title{
Caracterização dos Grupos de Pesquisa da Área da Educação Física do Brasil que Estudam a Deficiência
}

\author{
Characterization of research groups focused on disability in the area \\ of Physical Education in Brazil
}

\author{
TATTIANA CÔRTES FARIAS DE MENDONÇA \\ THIAGO FERREIRA DE SOUSA \\ CRISTIANO SANT'ANNA BAHIA \\ FRANCISCO TEIXEIRA COELHO
}

\section{RESUMO}

Objetivo: O objetivo deste estudo foi descrever a distribuição e as características dos grupos de pesquisa relacionados às pessoas com deficiência, na área de Educação Física no Brasil e a produção científica vinculada aos grupos. Material e Métodos: Foi realizado um estudo descritivo, de natureza documental, por meio da análise dos grupos de pesquisa cadastrados no Diretório de Grupos de Pesquisa do Conselho Nacional de Desenvolvimento Científico e Tecnológico (CNPq). A busca foi realizada em junho e julho de 2014 e os grupos identificados foram caracterizados e estratificados em específicos e não específicos para a deficiência, com base nas linhas de pesquisa. Nos grupos de pesquisa específicos, a produção dos líderes foi analisada quanto aos tipos de deficiência. Resultados: Foram encontrados 70 grupos de pesquisa, dos quais 38 foram incluídos, sendo 22 considerados específicos para as pessoas com deficiência. $\mathrm{O}$ artigo original foi a principal forma de veiculação das informações dos grupos de pesquisa específicos. A deficiência motora foi o tema predominante entre as investigações dos grupos de pesquisa específicos, perfazendo 75 publicações de artigos originais, seguido do assunto deficiência visual. Os grupos concentraram-se na região sudeste $(42 \%)$. Conclusão: Conclui-se que as investigações foram direcionadas para a deficiência motora e que 0 artigo original foi a principal modalidade de divulgação do conhecimento dos grupos específicos da deficiência. A quantidade de grupos específicos mostrou-se desigual entre as regiões brasileiras.

\section{DESCRITORES}

Grupos de Pesquisa. Pessoas com Deficiência. Indicadores de Produção Científica. Educação Física.

\begin{abstract}
Objective: To describe the distribution and characteristics of research groups focused on people with disabilities in the area of Physical Education in Brazil, as well as to describe their scientific production. Material and Methods: A descriptive study was conducted through electronic data analysis of the research groups registered in the Research Group Directory (Diretório de Grupos de Pesquisa) of the National Council for Scientific and Technological Development (Conselho Nacional de Desenvolvimento Científico e Tecnológico, CNPq). The searches were performed in June and July 2014, and the groups were characterized and further stratified as directly or indirectly related to disability based on their research lines. In the specific research groups, production of the group leaders was analyzed according to the types of disability. Results: A total of 70 research groups were found, of which 38 were included for analysis and 22 were considered to be specific for people with disabilities. Original article was the main form of propagation of information from specific research groups. Physical disabilities was the most frequent topic, totaling 75 publications of original articles, followed by visual impairment. The groups were mostly concentrated in southeastern Brazil $(42 \%)$. Conclusion: The research groups have mostly addressed motor disabilities, and original article was the main mechanism of disability-targeted groups for dissemination of knowledge. The amount of specific groups was found to be uneven across different regions.
\end{abstract}

\section{DESCRIPTORS}

Research Groups. Disabled Persons. Scientific Publication Indicators. Physical Education.

\footnotetext{
Supervisora da Fundação Regina Cunha. Itabuna. Bahia Brasil.

Docente do Departamento de Ciências do Esporte da Universidade Federal do Triângulo Mineiro. Uberaba. Minas Gerais. Brasil.

3 Docente do Departamento de Ciências da Saúde da Universidade Estadual de Santa Cruz. Ilhéus. Bahia. Brasil.

4 Docente do Centro de Formação de Professores da Universidade Federal do Recôncavo da Bahia. Amargosa. Bahia. Brasil.
} 
$\mathrm{O}$ s grupos de pesquisa (GP) de instituições de ensino superior, públicas e privadas, são responsáveis pela produção de conhecimento no Brasil. Os GP normalmente estão cadastrados no Diretório de Grupos de Pesquisa do Conselho Nacional de Desenvolvimento Científico e Tecnológico (CNPq) e discriminam as informações essenciais de seus grupos, como linhas de pesquisa, objetivo(s) de cada linha e os pesquisadores vinculados, sendo uma base essencial para a observação do perfil dos GP brasileiros.

Tendo em vista a relevância desse instrumento de consulta às informações dos GP, diferentes pesquisas foram conduzidas visando descrever o estado da arte dos temas priorizados pelos GP, para a produção do conhecimento, tais como o envelhecimento ${ }^{1}$ e a atividade física e saúde ${ }^{2}$. Dentre os objetos de estudo dos GP, citase, no cenário acadêmico científico, a deficiência como um de seus focos. Em estudo sobre os GP com o enfoque no lazer das pessoas com deficiência (PD) foram identificados 256 GP cadastrados, sendo que 73 foram específicos para essa temática ${ }^{3}$. Em outro estudo com enfoque na atividade física adaptada foram encontrados $23 \mathrm{GP}^{4}$.

Ressalta-se que o foco na produção de conhecimento sobre a deficiência mostra-se essencial, pois, de acordo com o censo demográfico do Brasil, realizado em 2010, mais de 45 milhões (aproximadamente 24\%) de brasileiros apresentavam pelo menos uma deficiência (visual, auditiva, motora ou intelectual) ${ }^{5}$. A deficiência pode ser entendida como a perda ou a anormalidade da estrutura ou função psicológica, fisiológica ou anatômica, de forma temporária ou permanente ${ }^{6}$. A Secretaria de Educação Especial do Ministério da Educação do Brasil conceitua PD como "aquelas que apresentam impedimentos em longo prazo, sendo essas limitações de ordem física, mental ou sensorial, que juntamente com a interação com outras barreiras podem minimizar a participação plena e efetiva em âmbito escolar e social"”.

Diante do cenário quantitativo de deficientes no Brasil, ações e políticas públicas com foco ao acesso à educação, atenção à saúde, inclusão social e acessibilidade ${ }^{8,9}$, com o intuito de melhoria das condições de vida dessas pessoas poderiam ser melhor aplicadas, por intermédio do uso de informações provenientes das pesquisas científicas conduzidas pelos GP ${ }^{10}$. Torna-se essencial, portanto, compreender o estado atual dos GP relacionados às $P D$ no Brasil. $O$ objetivo deste estudo foi descrever a distribuição e as características dos GP relacionados às PD, cadastrados no CNPq, na área de Educação Física no Brasil e a produção científica vinculada aos grupos.

\section{MATERIAL E MÉTODOS}

Este estudo caracteriza-se como descritivo, de natureza documental, mediante a análise de dados eletrônicos, obtidos na base de dados do Diretório de Grupos de Pesquisa do CNPq (http:// d g p . c n p q . br/dg p/f a c es / consulta/ consulta_parametrizada.jsf). A busca foi realizada em junho e julho de 2014. As expressões utilizadas para a consulta foram: deficiência, necessidades especiais, atividade motora adaptada, esporte adaptado, esporte paraolímpico, atividade física adaptada, educação física adaptada, educação especial, necessidades educacionais especiais, acessibilidade, inclusão. Adotou-se filtro para a grande área do grupo "Ciências da Saúde" e para a área do grupo "Educação Física". Os grupos identificados foram analisados individualmente e considerados elegíveis, caso apresentassem, pelo menos uma linha de pesquisa relacionada às $P D$ e não estivessem inativos.

Os grupos identificados foram caracterizados quanto ao nome do grupo, unidade da federação, nome da instituição, tipo da instituição (pública ou privada), ano de formação, número de pesquisadores (professores, estudantes e técnicos), titulação do líder do grupo e quantidade de linhas de pesquisa totais e específicas (relacionadas às PD). Posteriormente, eles foram considerados específicos ou não específicos.

Os específicos foram aqueles que apresentaram mais que $50 \%$ das linhas relacionadas às $\mathrm{PD}$ e os que apresentaram duas 
linhas, sendo uma delas relacionada às PD. Os grupos não específicos deveriam apresentar pelo menos uma linha de pesquisa relacionada às PD. Os que não corresponderam a este critério foram excluídos. Na ocorrência de dúvidas se a linha estava relacionada às PD foram analisadas as palavras-chave e o objetivo(s) da linha.

Para os grupos específicos, a produção dos líderes foi quantificada e apresentada individualmente, em relação à quantidade total e específica (enfoque sobre as PD). Para tanto, procedeu-se a leitura dos títulos das produções. Categorizaramse as produções específicas quanto ao tipo de deficiência: visual, auditiva, motora, intelectual, múltipla e não específica (quando não havia ênfase em determinada deficiência). As produções investigadas dos líderes foram: artigos publicados, artigos aceitos, livros e capítulos de livros. Obtiveram-se tais informações na plataforma Lattes (http://lattes.cnpq.br/), por meio do acesso ao currículo dos líderes. Os dados foram tabulados no software Excel (versão 2007) e empregadas às análises descritivas das frequências absolutas e relativas.

\section{RESULTADOS}

Encontraram-se 70 GP cadastrados, dos quais foram excluídos 18 que estavam inativos $\mathrm{e}$ 14 que não apresentavam nenhuma linha de pesquisa relacionada às PD. Ao final, 38 GP atenderam os critérios de inclusão, sendo 22 grupos específicos. Das 61 linhas de pesquisa dos GP específicos, 55 foram relacionadas à deficiência. $A$ Figura 1 apresenta a distribuição da quantidade de linhas totais e específicas em relação aos GP específicos e não específicos.

A Tabela 1 apresenta a distribuição dos GP em relação à região do Brasil, aos pesquisadores e ao tipo de instituição de ensino. Observou-se, de forma geral, nos grupos específicos e não específicos, a predominância na região sudeste do Brasil. A maioria dos grupos específicos estava composta por estudantes pertencentes às instituições de ensino públicas.

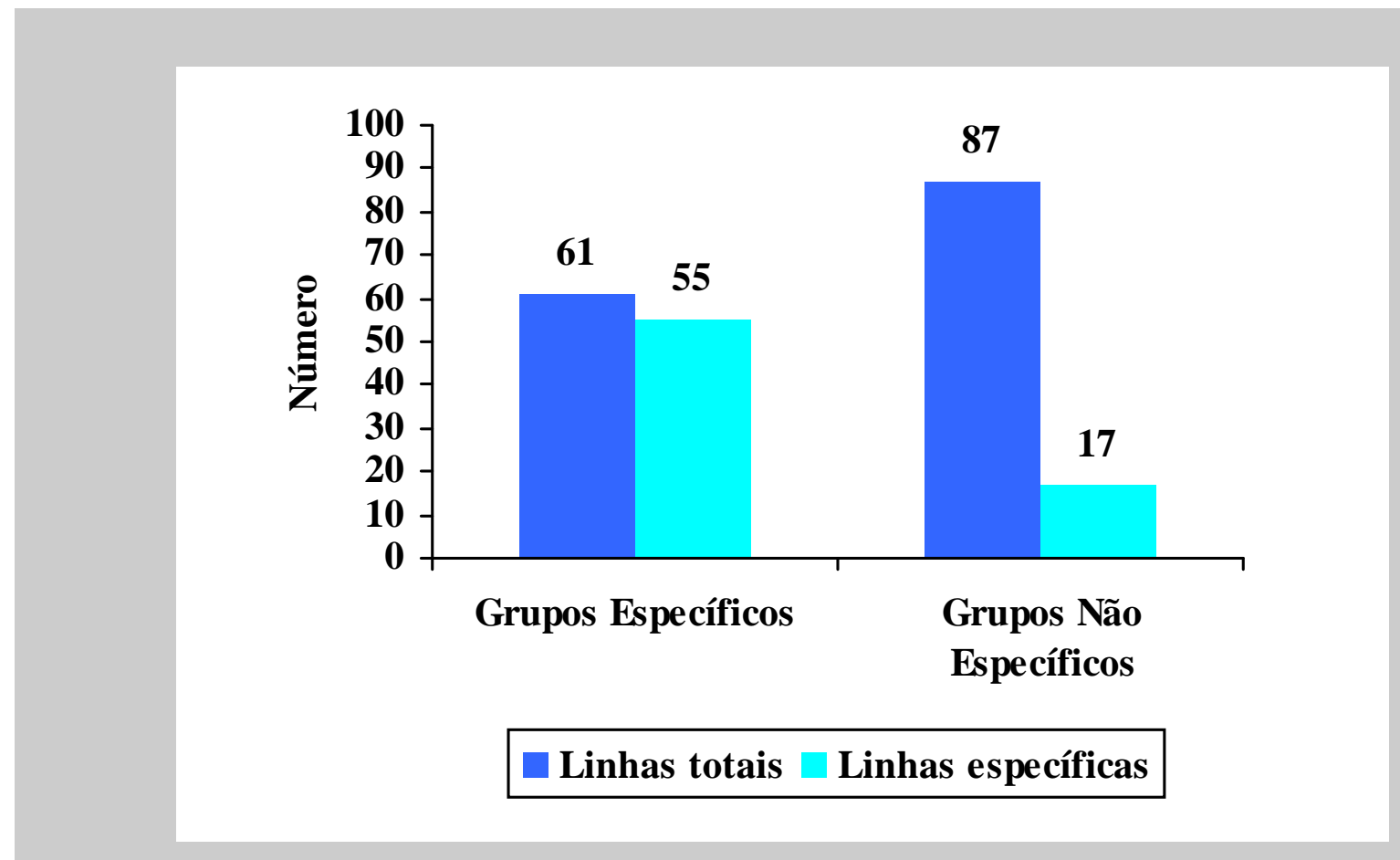

Figura 1. Distribuição das linhas de pesquisa totais e especíicicas de acordo com os grupos específicos e não específicos para o tema das pessoas com deficiência na área de Educação Física do Brasil, no ano de 2014. 


\begin{tabular}{|c|c|c|c|c|c|c|}
\hline \multirow{3}{*}{ Variáveis } & \multirow{2}{*}{\multicolumn{2}{|c|}{ Geral }} & \multirow{2}{*}{\multicolumn{2}{|c|}{ Grupos Especificos }} & \multirow{2}{*}{\multicolumn{2}{|c|}{ Grupos Não Específicos }} \\
\hline & & & & & & \\
\hline & $\mathbf{n}$ & $\%$ & $\mathbf{n}$ & $\%$ & $\mathbf{n}$ & $\%$ \\
\hline \multicolumn{7}{|l|}{ Região } \\
\hline Norte & 2 & 5.3 & 1 & 4.5 & 1 & 6.3 \\
\hline Nordeste & 7 & 18.4 & 4 & 18.2 & 3 & 18.8 \\
\hline Centro-oeste & 2 & 5.3 & - & - & 2 & 12,5 \\
\hline Sudeste & 16 & 42,1 & 10 & 45,5 & 6 & 37,4 \\
\hline Sul & 11 & 28.9 & 7 & 31.8 & 4 & 25.0 \\
\hline \multicolumn{7}{|l|}{ Pesquisadores } \\
\hline Professores & 275 & 42,2 & 124 & 35,3 & 151 & 50,3 \\
\hline Estudantes & 364 & 56.0 & 218 & 62,1 & 146 & 48.7 \\
\hline Técnicos & 12 & 1.8 & 9 & 2.6 & 3 & 1.0 \\
\hline \multicolumn{7}{|c|}{ Instituicão de ensino } \\
\hline Publica & 34 & 89.5 & 22 & 100.0 & 12 & 75.0 \\
\hline Privada & 4 & 10,5 & - & - & 4 & 25,0 \\
\hline
\end{tabular}

O primeiro grupo cadastrado data do ano de 1987, sendo caracterizado como não específico. Os primeiros grupos específicos foram cadastrados no ano 2000 (Figura 2). Desde 2008, houve crescimento da quantidade de grupos de pesquisa não específicos, seguido de seu declínio a partir de 2010. O maior pico ocorreu com os grupos específicos, com o aumento em 2011, mediante o cadastramento de quatro grupos.
Em apenas um grupo específico, o líder tinha titulação de mestre, sendo os demais doutores. Um pouco mais da metade dos artigos publicados são específicos, perfazendo 316 de um total de 562 (Tabela 2). A maioria das demais formas de produção revelou-se específica para a deficiência. Nas produções de artigos, foi dada maior ênfase às deficiências motora e visual (Tabela 3). $O$ Grupo de Pesquisa em Avaliação Motora Adaptada da

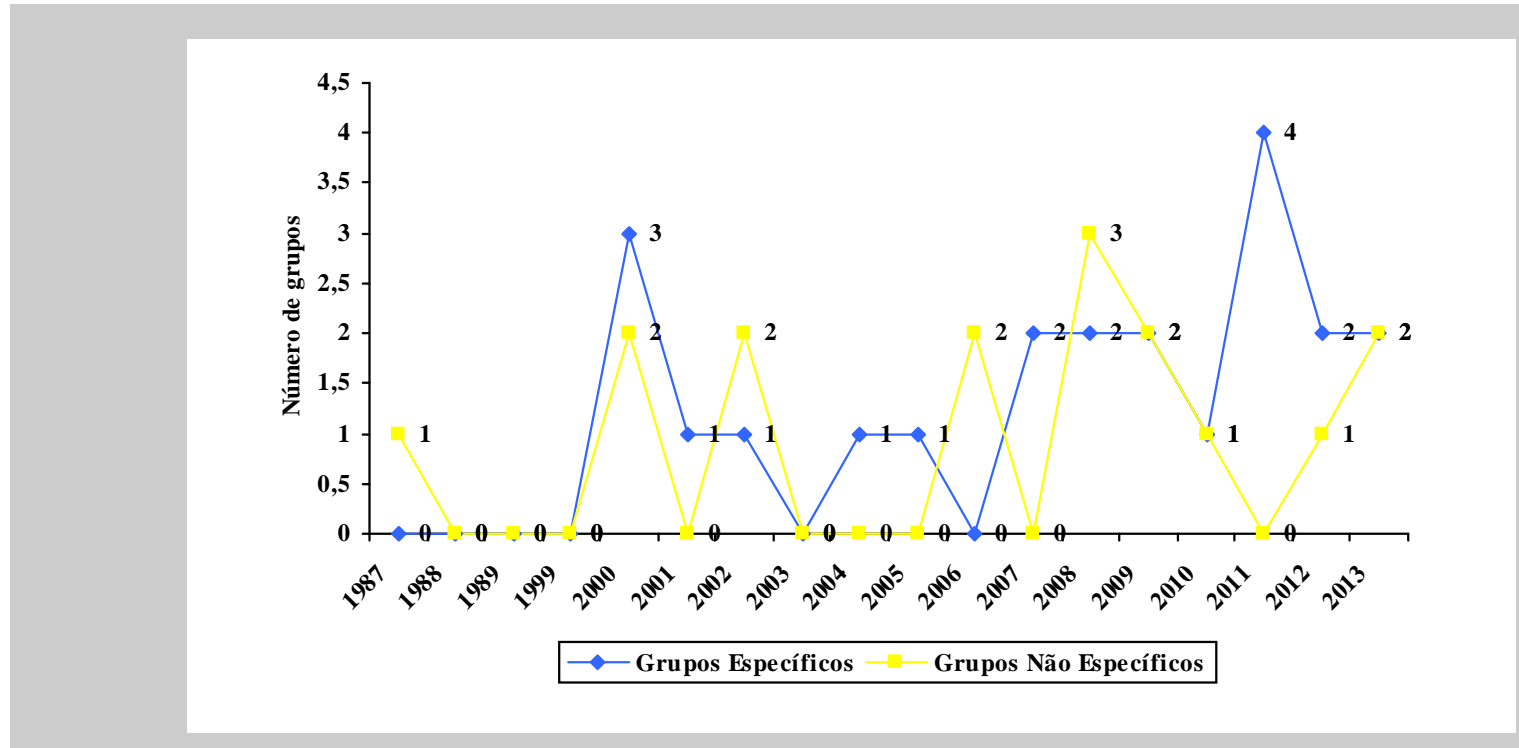

Figura 2. Quantidade de grupos de pesquisa específicos e não específicos para o tema das pessoas com deficiência na área de Educação Física do Brasil, de acordo com o ano de cadastramento no CNPq, no ano de 2014. 
Tabela 2. Distribuição da publicação dos líderes dos grupos de pesquisa específicos para as pessoas com deficiência na área de Educação Física no Brasil, de acordo com o tipo de publicação Grupos de Pesquisa

Atividade Motora Adaptada - UFSC

Atividade Fisica Adaptada - UFPB

Estudos sobre Pessoas com Deficiências e Atividades

Motoras - UFAM

Grupo de Estudo e Pesquisa em Atividade Física e

Deficiência - UEL

Grupo de Estudo e Pesquisa em Educação Fisica

Deficiência, Inclusão e Escola - UNICAMP

Grupo de Estudos e Extensåo em Atividade Motora Adaptada

- UFAL

Grupo de Estudos e Pesquisa em Atividade Física Adaptada

na Unioeste - UNIOESTE

Grupo de Estudos e Pesquisa em Esportes e Deficiência

Visual - UNICAMP

Grupo de Pesquisa em Avaliação Motora Adaptada-

UNICAMP

Grupo de Pesquisa em Inclusāo, Movimento e Ensino a

Distância - UFJF

Grupo de Pesquisas e Estudos em Acessibilidade e Inclusão Sustentável - UFF

Núcleo de Estudos em Atividade Fisica Adaptada - UFSCAR

Núcleo de Educacão Física e Esportes Adaptados - UEFS

Tecnologias Assistivas no Esporte, Lazer e Saúde - IFES

Avaliaçōes e Intervençōes Motoras - UFRGS

Formação Profissional em Contextos Educacionais Inclusivos

- UNICENTRO

Grupo de Estudos e Pesquisa em Imagem Corporal -

UNICAMP

Atividade Motora Adaptada - UDESC

Centro de Estudos Olimpicos e Paraolímpicos - UFRGS

Grupo de Estudos em Atividade Física e Saúde - UFSJ

Grupo de Estudos, Intervenção e Pesquisa em Educaçāo

Escolar e Teoria Historico Cultural - UNESP

Grupo de Pesquisa em Desenvolvimento e Aprendizagem

Motora - UFRB

Total e diferenciação em quantidade total e específica para a deficiência, no ano de 2014.

\begin{tabular}{|c|c|c|c|c|c|c|c|}
\hline $\begin{array}{l}\text { Artigos no } \\
\text { total }\end{array}$ & $\begin{array}{l}\text { Artigos } \\
\text { específicos }\end{array}$ & $\begin{array}{l}\text { Artigos } \\
\text { aceitos no } \\
\text { total }\end{array}$ & $\begin{array}{l}\text { Artigos aceitos } \\
\text { específicos }\end{array}$ & $\begin{array}{l}\text { Livros no } \\
\text { total }\end{array}$ & $\begin{array}{c}\text { Livros } \\
\text { específicos }\end{array}$ & $\begin{array}{l}\text { Capítulos de } \\
\text { livros no total }\end{array}$ & $\begin{array}{l}\text { Capítulos de } \\
\text { livros } \\
\text { específicos }\end{array}$ \\
\hline 21 & 17 & - & - & - & - & 1 & 1 \\
\hline - & - & - & - & - & - & 1 & 1 \\
\hline 15 & 9 & - & - & 2 & 2 & 3 & 3 \\
\hline 29 & 21 & 3 & 3 & 4 & 4 & 7 & 7 \\
\hline 39 & 34 & 1 & 1 & 11 & 11 & 18 & 17 \\
\hline 7 & 6 & - & - & 11 & 2 & 22 & 16 \\
\hline 20 & 15 & - & - & - & - & 8 & 7 \\
\hline 47 & 36 & 1 & 1 & 1 & 1 & 19 & 19 \\
\hline 69 & 62 & 5 & 3 & 9 & 9 & 24 & 24 \\
\hline 23 & 12 & 6 & 3 & 33 & 32 & 16 & 13 \\
\hline 40 & 21 & 1 & 1 & - & - & 2 & 1 \\
\hline 14 & 12 & 1 & - & 1 & 1 & 14 & 12 \\
\hline 6 & 5 & - & - & 1 & - & 2 & 1 \\
\hline 13 & 4 & - & - & 2 & - & - & - \\
\hline 60 & 3 & 1 & - & 1 & - & 10 & - \\
\hline 23 & 18 & 4 & 3 & 2 & 2 & 15 & 12 \\
\hline 63 & 20 & 2 & - & 4 & - & 16 & - \\
\hline 36 & 13 & 3 & 2 & 1 & - & 1 & - \\
\hline 7 & - & 1 & - & 5 & - & 19 & - \\
\hline 8 & 1 & - & - & $\cdot$ & - & - & - \\
\hline 15 & 4 & - & - & 2 & - & 21 & 1 \\
\hline 7 & 3 & - & - & - & - & 3 & 1 \\
\hline 562 & 316 & 29 & 17 & 90 & 64 & 222 & 139 \\
\hline
\end{tabular}

Tabela 3. Distribuição da produção de artigos publicados e aceitos específicos para as pessoas com deficiência, dos líderes dos grupos de pesquisa específicos na área de Educação Física no Brasil, de acordo com tipo de deficiência, no ano de 2014.

Grupos de Pesquisa

Atividade Motora Adaptada - UFSC

Atividade Fisica Adaptada - UFPB

Estudos sobre Pessoas com Deficiências e Atividades Motoras - UFAM

Grupo de Estudo e Pesquisa em Atividade Física e Deficiência - UEL

Grupo de Estudo e Pesquisa em Educação Física, Deficiência, Inclusão e Escola -

UNICAMP

Grupo de Estudos e Extensão em Atividade Motora Adaptada - UFAL

Grupo de Estudos e Pesquisa em Atividade Física Adaptada - UNIOESTE

Grupo de Estudos e Pesquisa em Esportes e Deficiência Visual - UNICAMP

Grupo de Pesquisa em Avaliacão Motora Adaptada - UNICAMP

Grupo de Pesquisa em Inclusão, Movimento e Ensino a Distância - UFJF

Grupo de Pesquisas e Estudos em Acessibilidade e Inclusão Sustentável - UFF

Núcleo de Estudos em Atividade Física Adaptada - UFSCAR

Núcleo de Educação Física e Esportes Adaptados - UEFS

Tecnologias Assistivas no Esporte, Lazer e Saúde - IFES

Avaliacōes e Intervencōes Motoras - UFRGS

Formação Profissional em Contextos Educacionais Inclusivos - UNICENTRO

Grupo de Estudos e Pesquisa em Imaqem Corporal - UNICAMP

Atividade Motora Adaptada - UDESC

Centro de Estudos Olímpicos e Paraolímpicos - UFRGS

Grupo de Estudos em Atividade Física e Saúde - UFS.J

Grupo de Estudos, Intervenção e Pesquisa em Educação Escolar e Teoria

Histórico Cultural - UNESP

Grupo de Pesquisa em Desenvolvimento e Aprendizagem Motora - UFRB

Total Artigos

Artigos aceitos

DV: deficiência visual; DA: deficiência auditiva; DM: deficiência motora; DI: deficiência intelectual; DMu: deficiência múltipla; NE: não especifica. 
UNICAMP foi o que mais produziu artigos com foco na deficiência motora e o Grupo de Estudos e Pesquisa em Esportes e Deficiência Visual (UNICAMP) foi o que mais produziu artigos com foco na deficiência visual. No entanto, em 122 publicações originais não foi possível discriminar a ênfase em determinada deficiência.

Em relação à produção de livros (Tabela 4), sobressaiu a ênfase na deficiência motora. Nos capítulos de livros, foi observado o enfoque nas deficiências motora e visual. Não foi detectado nenhum livro relacionado à deficiência auditiva. Percebeu-se também a ausência de capítulos de livros relacionados à deficiência múltipla. Em 124 publicações relativas a livros e a capítulos de livros, não foi possível identificar ênfase em determinado tipo de deficiência.

\section{DISCUSSÃO}

No presente estudo, a quantidade de GP relacionados às $\mathrm{PD}$ foi semelhante ao estudo que com enfoque na temática da atividade física adaptada, no entanto, sem restrição para a área de pesquisa ${ }^{4}$. As principais diferenças entre os estudos estão no método, pois neste estudo optou-se por adotar diferentes termos/descritores, a não inclusão de grupos inativos no período da coleta de dados, definição de grupos como específicos, levando em consideração as linhas de pesquisa e o direcionamento para área da Educação Física.

Em relação a esse quantitativo de GP na área da Educação Física, é importante considerar a inclusão desse tema como disciplina nos currículos dos cursos de licenciatura em Educação Física, conforme a Resolução de № 03 de $1987^{11}$. Além disso, a portaria oํ 1.793, de dezembro de $1994^{12}$, destacou a necessidade de inclusão da disciplina "aspectos ético-político educacionais da normalização e integração da pessoa portadora de necessidades especiais", nos cursos de licenciatura e da área da saúde, dentre eles a Educação Física. Essas ações representaram componentes estimuladores da discussão e, consequentemente, do desenvolvimento de pesquisas referentes a temas envolvendo PD.

Na caracterização dos grupos por região, a sudeste apresentou predomínio dos GP específicos. Essa concentração pode estar

\begin{tabular}{|c|c|c|c|c|c|c|c|c|c|c|c|c|}
\hline \multirow{2}{*}{ Grupos de Pesquisa } & \multicolumn{6}{|c|}{ Livros } & \multicolumn{6}{|c|}{ Capitulos de livros } \\
\hline & DV & DA & DM & DI & DMu & NE & DV & DA & DM & DI & DMu & NE \\
\hline Atividade Motora Adaptada - UFSC & - & - & - & - & - & - & - & - & - & - & - & 1 \\
\hline Atividade Física Adaptada - UFPB & - & - & - & - & - & - & - & - & - & - & - & 1 \\
\hline Estudos sobre Pessoas com Deficiências e Atividades Motoras - UFAM & - & - & 1 & - & - & 1 & - & - & - & - & - & 3 \\
\hline Grupo de Estudo e Pesquisa em Atividade Física e Deficiência - UEL & - & - & - & - & - & 4 & 1 & 1 & 1 & 1 & - & 3 \\
\hline $\begin{array}{l}\text { Grupo de Estudo e Pesquisa em Educação Física, Deficiência, Inclusāo e Escola - } \\
\text { UNICAMP }\end{array}$ & - & - & 2 & - & - & 9 & - & 2 & 6 & - & - & 9 \\
\hline Grupo de Estudos e Extensão em Atividade Motora Adaptada - UFAL & - & - & - & 1 & - & 1 & 1 & 1 & - & 2 & - & 12 \\
\hline Grupo de Estudos e Pesquisa em Atividade Física Adaptada - UNIOESTE & - & - & - & - & - & - & - & - & 2 & - & - & 5 \\
\hline Grupo de Estudos e Pesquisa em Esportes e Deficiência Visual - UNICAMP & 1 & - & - & - & - & - & 13 & - & - & - & - & 6 \\
\hline Grupo de Pesquisa em Avaliacāo Motora Adaptada - UNICAMP & - & - & 2 & - & - & 7 & - & - & 15 & - & - & 9 \\
\hline Grupo de Pesquisa em Inclusão, Movimento e Ensino a Distância - UFJF & - & - & 6 & - & - & 26 & - & - & 8 & 1 & - & 4 \\
\hline $\begin{array}{l}\text { Grupo de Pesquisas e Estudos em Acessibilidade e Inclusão Sustentável - } \\
\text { UFF }\end{array}$ & - & - & - & - & - & - & - & - & - & - & - & 1 \\
\hline Núcleo de Estudos em Atividade Fisica Adaptada - UFSCAR & 1 & - & - & - & - & - & 6 & - & - & 1 & - & 5 \\
\hline Núcleo de Educacão Física e Esportes Adaptados - UEFS & - & - & - & - & - & - & - & - & - & - & - & 1 \\
\hline Tecnologias Assistivas no Esporte, Lazer e Saúde - IFES & - & - & - & - & - & - & - & - & - & - & - & - \\
\hline Avaliaç̃es e Intervenç̃es Motoras - UFRGS & - & - & - & - & - & - & - & - & - & - & - & - \\
\hline Formação Profissional em Contextos Educacionais Inclusivos - UNICENTRO & - & - & - & - & - & 2 & - & - & - & 1 & - & 11 \\
\hline Grupo de Estudos e Pesquisa em Imagem Corporal - UNICAMP & - & - & - & - & - & - & - & - & 2 & - & $=$ & 1 \\
\hline Atividade Motora Adaptada - UDESC & - & - & - & - & - & - & - & - & - & - & - & - \\
\hline Centro de Estudos Olimpicos e Paraolimpicos - UFRGS & - & - & - & - & - & - & - & - & - & - & - & - \\
\hline Grupo de Estudos em Atividade Física e Saúde - UFSJ & - & - & - & - & - & - & - & - & - & - & - & - \\
\hline $\begin{array}{l}\text { Grupo de Estudos, Intervenção e Pesquisa em Educação Escolar e Teoria } \\
\text { Histórico Cultural - UNESP }\end{array}$ & - & - & - & - & - & - & - & - & - & - & - & 1 \\
\hline Grupo de Pesquisa em Desenvolvimento e Aprendizaqem Motora - UFRB & - & - & - & - & - & - & - & - & - & - & - & 1 \\
\hline Total & 2 & - & 11 & 1 & 0 & 50 & 21 & 4 & 34 & 6 & - & 74 \\
\hline
\end{tabular}

DV: deficiência visual; DA: deficiência auditiva; DM: deficiência motora; DI: deficiência intelectual; DMu: deficiência múltipla; NE: não especifica. 
relacionada a quantidade de programas de pósgraduação em Educação Física nessa região, pois, no Brasil, os GP normalmente apresentam relação com os programas de formação stricto sensu. A distribuição desproporcional dos grupos, entre as regiões do país, foi encontrada em outras pesquisas sobre os $\mathrm{GP}^{1-3}$. No entanto, as maiores taxas de pessoas com no mínimo uma deficiência, de acordo com o Censo de 2010, estão na região nordeste ${ }^{5}$, 0 que remete a necessidade de ampliação do escopo de ações de GP dessa região para as PD.

No presente estudo, todos os GP específicos estavam nas instituições de ensino superior pública. Essas informações corroboram com outros estudos com foco nos GP relacionados às $\mathrm{PD}^{3,4}$. As universidades e os centros de ensino superior públicos apresentam maior perfil para a formação de GP, os quais, com frequência, dão sustentabilidade aos programas de pós-graduação, especificamente devido à forte ênfase na consolidação do tripé ensino-pesquisa-extensão ${ }^{13}$; regime de carga horária de trabalho ${ }^{14}$; e financiamento de bolsas de pesquisa ${ }^{15}$.

A quantidade de GP específicos mantevese estável entre os anos de 2001 e 2010, com maior pico no ano de 2011. É relevante considerar que o aumento dos GP, no Brasil, ocorreu também em outras áreas de estudo, conforme verificado nas pesquisas sobre os GP com ênfase no envelhecimento ${ }^{1}$ e na atividade física e saúde ${ }^{2}$. O aumento de GP específicos pode ter ocorrido em virtude da valorização das PD e de seu reconhecimento pela sociedade após o crescimento da discussão sobre o tema ${ }^{16,17}$. O primeiro GP cadastrado no diretório surgiu no ano de 1987, e nesse período houve a implantação do ideal de integração dos deficientes ${ }^{18}$. Em anos posteriores, outros aportes teóricos ${ }^{7,13,14,19-26}$ contribuíram no aspecto legislativo e de consolidação de elementos para as PD, o que talvez também tenha favorecido o surgimento de mais GP, tendo em vista a criação de no mínimo um grupo por ano, devido à necessidade de investigação do impacto desses elementos na sociedade.

As produções de artigos, livros e capítulos de livros descritas neste estudo mostraram maior ênfase na deficiência motora. $O$ Grupo de Pesquisa em Avaliação Motora Adaptada (UNICAMP) apresentou discrepância na quantidade de artigos relacionados à deficiência motora, o que favoreceu a expressiva quantidade para esse tipo de deficiência. O perfil de produção para essa temática está pautado, principalmente, pelo objeto de estudo da Educação Física: a cultura corporal27. Tal produção busca desenvolver uma reflexão pedagógica sobre o acervo de formas de representação do mundo que o homem tem produzido, no decorrer da história, exteriorizadas pela expressão corporal: jogos, danças, lutas, ginástica, esportes e outros ${ }^{27}$. De acordo com o censo de 2010, a segunda principal deficiência detectada foi a motora, que abrangeu $7 \%$ da população, especialmente, pessoas de 65 anos ou mais (38,3\%), o que evidencia o processo de envelhecimento e a consequente perda da autonomia funcional aliados da deficiência motora ${ }^{5}$. Essa característica demográfica pode justificar tanto a quantidade de trabalhos produzidos sobre a deficiência motora quanto à quantidade de grupos sobre o envelhecimento ${ }^{1}$.

A segunda principal temática dos artigos publicados pelos GP foi a deficiência visual. De acordo com o censo de 2010, considerando o grau de severidade da deficiência e a autopercepção da pessoa sobre suas funcionalidades, a deficiência visual apresentou a maior ocorrência no país e afetou $18,6 \%$ da população brasileira, de forma predominante em mulheres, sendo, porém, prevalente em todas as faixas etárias e de forma mais acentuada no grupo com 65 anos ou mais $(49,8 \%)^{5}$. Por representar o tipo de deficiência que mais afetou a população brasileira, conforme o Censo de 2010, a ocorrência de pesquisas com ênfase nessa deficiência pode ser mais enfatizada. 
Araújo $^{28}$ mostrou que a Paralimpíada de 1976, realizada em Toronto, marcou a inclusão dos atletas com deficiência visual no cenário internacional esportivo. Nesses jogos, houve participação de 1.657 atletas, sendo 187 atletas com deficiência visual, algo relevante para a área esportiva e científica ${ }^{29}$. Desde então, novas modalidades foram se adequando às especificidades deste grupo, que atualmente disputa 11 das 22 modalidades paralímpicas. A produção acadêmica abordando esse tipo de deficiência pode, portanto, relacionar-se com a área esportiva, em virtude das modalidades paralímpicas, que se expandiram nos últimos anos ${ }^{30}$.

Algumas limitações do presente estudo referem-se ao período de levantamento das informações - junho e julho de 2014 - pois, outros grupos podem ter surgido posteriormente, bem como a possibilidade de ter sido alterado o perfil das produções realizadas. Outras se referem à consideração da produção do líder do grupo, como representativa do grupo, porque pode, parcialmente, não caracterizar o foco da produção dos grupos específicos. Ressalva-se que este estudo trata do direcionamento da produção dos grupos específicos sobre as PD na área da Educação Física.

\section{REFERÊNCIAS}

1. Borges LJ, Santos SFS, Scherer FC, Benedetti TRB. Grupos de pesquisa sobre atividade física e envelhecimento no Brasil. Rev. Bras. Ativ. Fís. Saúde. 2012;17(2):114-20.

2. Santos A, Bastos LLAG, Aleixo AA, Paulo TRS, Mendes EL. Distribuição, evolução e produção científica dos grupos de pesquisa em atividade física e saúde do Brasil. Rev. Bras. Ativ. Fís. Saúde. 2012;4(17):258-62.

3. Silva JVP, Mendonça TCF, Sampaio TMV. Grupos de pesquisas e enfoque dado ao lazer das pessoas com deficiência na produção científica no Brasil. Licere. 2014;17(3):66-98.

\section{CONCLUSÃO}

Conclui-se que as produções tiveram maior foco na deficiência motora. $\mathrm{O}$ artigo original representou a principal forma de veiculação do conhecimento dos grupos específicos. A quantidade de grupos específicos com ênfase na deficiência foi desigual nas regiões brasileiras. Houve estabilização da quantidade de grupos cadastrados ao longo dos anos. Sugere-se, em futuras pesquisas, o detalhamento das produções relacionadas à deficiência, quanto aos tipos de estudo, às subáreas investigadas e à classificação dos periódicos dos artigos publicados.

Os dados encontrados, neste estudo, possibilitaram a caracterização da distribuição dos grupos específicos da área de Educação Física no Brasil, assim como o conhecimento do estado atual sobre a significância dessa temática para a área. Diante da realidade encontrada e da relevância da pesquisa para a proposição de ações, de políticas públicas e de outros tipos de mecanismos indutores de práticas para a comunidade, a presente investigação mostra-se muito importante, por sua contribuição para a concretização de melhorias que favoreçam PD.
4. Munster MA, Rossi P, Fernandes EF. Diretório dos Grupos de Pesquisa do CNPq: análise da produção científica em atividade física adaptada. Rev. Soci. Bras. Ativ. Mot. Adap. 2012;13(2):18-24.

5. Instituto Brasileiro de Geografia e Estatística. Características gerais da população, religião e pessoas com deficiência. 2010. Disponível em: <http:// biblioteca.ibge.gov.br/visualizacao/periodicos/94/ cd_2010_religiao_deficiencia.pdf>. Acesso em: 27 de abr. 2015.

6. Amiralian MLT, Pinto EB, Ghirardi MIG, Lichtig I, Masini EFS, Pasqualin L. Conceituando a deficiência. Rev. Saúde Pública. 2000;34(1):97-103. 
7. Ministério da Educação do Brasil. Política Nacional de Educação Especial na Perspectiva da Educação Inclusiva. Brasília, DF, 2007. Disponível em: <http:// p e e i.me c.gov. br/arquivos/ politica_nacional_educacao_especial.pdf $>$. Acesso em: 27 de abr. 2015.

8. República Federal do Brasil. Decreto № 7.612 de 17 de novembro de 2011. Presidência da República. Brasília, DF, 2011. Disponível em: <http://www.planalto.gov.br/ ccivil_03/_Ato2011-2014/2011/Decreto/D7612.htm>. Acesso em: 27 de abr. 2015.

9. República Federativa do Brasil. Lei n. 7.853 de 24 de outubro de 1989. Presidência da República. Brasília, DF, 1989. Disponível em: <http://www.planalto.gov.br/ ccivil_03/Leis/L7853.htm>. Acesso em: 27 de abr. 2015.

10. Gil, AC. Como elaborar projetos de pesquisa. São Paulo: Atlas; 2002.

11. Ministério da Educação e Cultura do Brasil. Resolução nº 03, de 16 de Junho de 1987. Brasília, DF, 1987. Disponível em: <http://crefrs.org.br/legislacao/pdf/ resol_cfe_3_1987.pdf>. Acesso em: 31 de jan. 2015.

12. Ministério da Educação e Cultura do Brasil. Portaria N. 1.793, de dezembro de 1994. Brasília, DF, 1994. Disponível em: <http://portal.mec.gov.br/seesp/ arquivos/pdf/port1793.pdf>. Acesso em: 27 de abr. 2015.

13. República Federativa do Brasil. Constituição da República Federativa do Brasil, 1988. Brasília, DF, 1988. Disponível em: <http://www.planalto.gov.br/ccivil_03/ Constituicao/Constituicao.htm>. Acesso em: 27 de abr. 2015.

14. República Federativa do Brasil. Decreto n. 3.076, de 1ำ de junho de 1999. Presidência da República. Brasília, DF, 1999. Disponível em: <http://www.planalto.gov.br/ ccivil_03/decreto/D3076.htm>. Acesso em: 27 de abr. 2015.

15. Geocapes. Dados Estatísticos. 2013. Disponível em: <http://geocapes.capes.gov.br/geocapes2/>. Acesso em: 14 de maio 2015.

16. Secretaria dos Direitos das Pessoas com Deficiência. 30 anos do AIPD: Ano Internacional das Pessoas Deficientes 1981-2011. Memorial da Inclusão. São Paulo, 2011. Disponível em: < <http:// pessoacomdeficiencia.sp.gov.br/usr/share/documents/ 30\%20ANOS\%20AIPD\%20LIVRO\%20DIGITAL.pdf>. Acesso em: 27 de abr 2015.
17. República Federativa do Brasil. Decreto n. 93.481, de 29 de outubro de 1986. Presidência da República. Brasília, DF, 1999. Disponível em: <http:// www.planalto.gov.br/ccivil_03/decreto/1980-1989/ 1985-1987/D93481.htm>. Acesso em: 27 de abr. 2015.

18. Stainback W, Stainback S. Colaboração, Rede de apoio e construção de comunidade. In: Stainback W, Stainback S. Inclusão: um guia para educadores. Porto Alegre: Artes Médicas; 1999.

19. Sassaki RK. Inclusão: construindo uma sociedade para todos. Rio de Janeiro: WVA; 2004.

20. República Federativa do Brasil. Lei n. 8.069, de 13 de julho de 1990. Presidência da República. Brasília, DF, 1990. Disponível em: <http://www.planalto.gov.br/ ccivil_03/LEIS/L8069.htm>. Acesso em: 27 de abr. 2015.

21. Organização das Nações Unidas para a Educação, a Ciência e a Cultura. Declaração Mundial sobre Educação Para Todos (Conferência de Jomtien). Tailândia: Unesco, 1990. Disponível em: <http:// unesdoc.unesco.org/images/0008/000862/ 086291 por.pdf>. Acesso em: 27 de abr. 2015.

22. Ministério da Educação do Brasil. Declaração de Salamanca sobre princípios, políticas e práticas na área das necessidades educativas especiais. Salamanca, Espanha: UNESCO, 1994. Disponível em: <http:// portal.mec.gov.br/seesp/arquivos/pdf/salama nca.pdf>. Acesso em: 27 de abr. 2015.

23. República Federativa do Brasil. Lei n. 9.394/96 de 20 de dezembro de 1996. Presidência da República. Brasília, DF, 1996. Disponível em: <http:// www.planalto.gov.br/ccivil_03/Leis/L9394.htm >. Acesso em: 27 de abr. 2015.

24. Chicon JF, Soares JA. Compreendendo os conceitos de integração e inclusão. In: Chicon, JF. Educação especial: fundamentos para a prática pedagógica. Vitória, Edufes; 2004. p. 33-50.

25. Secretaria Especial dos Direitos Humanos do Brasil. A Convenção sobre Direitos das Pessoas com Deficiência Comentada. Ana Paula CR, Flavia Maria PV (Org.) Brasília: Secretaria Especial dos Direitos Humanos. Coordenadoria Nacional para Integração da Pessoa Portadora de Deficiência, 2008.

26. República Federativa do Brasil. Decreto n. 6.214, de 26 de setembro de 2007. Presidência da República. Brasília, DF, 2007. Disponível em: <http:// www.planalto.gov.br/ccivil_03/_Ato2007-2010/2007/ Decreto/D6214.htm>. Acesso em: 27 de abr. 2015. 
27. Coletivo de Autores. Metodologia do Ensino de Educação Física. São Paulo: Cortez; 1992.

28. Araújo, PF. Desporto Adaptado no Brasil: origem, institucionalização e atualidades. Tese [Doutorado em Educação Física Atividade Física e Adaptação] Universidade Estadual de Campinas; 1997.

29. Portal Oficial do Governo Federal sobre os Jogos Olímpicos e Paraolímpicos de 2016. Edições dos Jogos Paraolímpicos. Disponível em: <http:// www.brasil2016.gov.br/pt-br/paraolimpiadas/asedicoes>. Acesso em: 27 de abr 2015.

30. Comitê Paralímpico Brasileiro. Modalidades. Disponível em: <http://www.cpb.org.br/modalidades/>. Acesso em: 27 de abr 2015.

\section{CORRESPONDÊNCIA}

Thiago Ferreira de Sousa

Universidade Estadual de Santa Cruz

Departamento de Ciências da Saúde, Grupo de Pesquisa em Atividade Física e Saúde.

Campus Soane Nazaré de Andrade, km 16.

Rodovia Jorge Amado.

CEP 45662-900.- Ilhéus - Bahia - Brasil

Email: tfsousa_thiago@yahoo.com.br 